\title{
Stressors Perceived by the Para-clinical Undergraduate Medical Students
}

\author{
Brig Gen Md Ahsan Habib ${ }^{1}$, Lt Col Md Azizur Rahman ${ }^{2}$ Lt Col Amju Manara ${ }^{3}$, Lt Col Mahmuda Ayub ${ }^{4}$, Lt Col Nasrin \\ Begum $^{5}$, Lt Col Sharmin Hossain ${ }^{6}$
}

\begin{abstract}
Medical curricula are considered as toughest of all curricula of undergraduate professionals. Student faces many stress provoking factors in the academic course. In time identification and adoption of coping strategy can ensure proper achievement of goal of the curriculum. The objective of this prospective study was to find out the nature and intensity of stressors perceived by the mid level medical students (phase II \& III) before their summative examination. Regularly passed students of phase II and III undergraduate students of Armed Forces Medical College, Bangladesh were included in the study and the responses were collected in the first week of April 2018 (3 weeks prior to beginning of summative examination). Validated structured set of questionnaire (Medical Student Stressor Questionnaire -MSSQ) was selected for the study and was distributed to the volunteers of target population only. Falling behind in reading schedule, getting poor marks, facing illness or death of the patients and too much restriction in campus were identified as high intensity stressors by the phase II students. On the other hand high workload, not enough scope of medical skill practice, facing illness or death of the patients and too much restriction in campus were identified as high intensity stressors by the phase III students. Intensity of stressors was significantly higher in phase II students than phase III $(\mathrm{p}=0.000)$. This study focused the present status of an area. Identification and incorporation of strategies to improve the teaching, learning, evaluation and educational environment are required to help the students to develop stress coping skills in early medical career in order to reduce negative effects of stressors on the future doctors.
\end{abstract}

Key words: Stressor, undergraduate medical, questionnaire

\section{Introduction}

Optimal way to teach future generation is yet in dispute. Various disciplines are advancing in their own way to have undergraduate doctors, resulting in competitive overloading of the course content. The pressure among the students for achieving objectives in a limited time, as well as, to go ahead leaving other behind to be an idol of the time automatically create stress in the learners. Modern medical curricula are designed to foster learner engagement and to encourage

\footnotetext{
1. Brig Gen Md Ahsan Habib, MBBS, M Phil, MMEd

Professor, Department of Anatomy

Armed Forces Medical College, Dhaka

2. Lt Col Md Azizur Rahman, MBBS, MPhil

Associate Professor, Department of Anatomy

Armed Forces Medical College, Dhaka

3. Lt Col Amju Manara, MBBS, MS

Associate Professor, Department of Anatomy

Armed Forces Medical College, Dhaka

4. Lt Col Mahmuda Ayub, MBBS, M Phil

Associate Professor, Department of Anatomy

Armed Forces Medical College, Dhaka

5. Lt Col Nasrin Begum, MBBS, M Phil

Associate Professor, Department of Anatomy

Armed Forces Medical College, Dhaka

6. Lt Col Sharmin Hossain, MBBS, M Phil

Associate Professor, Department of Anatomy, Embryologist,

Fertility Centre, CMH Dhaka.
}

Address of correspondence: Brig Gen Md Ahsan Habib Professor and Head, Department of Anatomy, Armed Forces

Medical College, Dhaka Cantonment, Dhaka, Bangladesh

E-mail: doctorahsan@gmail.com active participation and to promote life-long learning. But, simultaneously the curriculum is failing to reduce the pressure of summative evaluation of students in undergraduate course.

The stress is the body's nonspecific response or reaction to demands made on it or to the disturbing events in the environment. It is not just a stimulus or a response but it is a process by which we perceive and cope with environmental threats and challenges'. Major stress factors of students identified through research as contributing to anxiety were excessive course load, lack of time to revise before examination and lack of systematic studies, parental expectations and lack of time for physical activity and extracurricular activities ${ }^{2}$. Excessive alertness to a stressful situation is harmful to students' performance and this leads to panic, sleeping disorders and depression.

As the financial reward is supposedly better, medical carrier is still at the height of its popularity. In Bangladesh through a tough competitive process, the students get in the course with full enthusiasm. Most of the students can continue with the same interest; but handsome number loses that the middle of the course. In some cases stress can not promote and facilitate learning and they gradually loose attention. Reduced concentration compels them to reduce self esteem and encourage them to adopt academic dishonesty. Emotional reactions continue and lead to low academic achievement and psychological distress. If the process is identified early the unfavourable stressor can be shifted to favourable one and the process of demotivation can be reversed.

Under graduate course of Bangladesh has four phases and

Bangladesh Journal of Medical Education 2018;9(2):3-10. C 2018 Habib et al., publisher and licensee Association for Medical Education. This is an Open Access article which permits unrestricted non-commercial use, provided the original work is properly cited. 
each phase ends with a university level summative test ${ }^{3}$. As this examination matter for promotion to the next phase; anxiety related to this examination is usually considered as the most stressing problem perceived by a student. This prospective study was designed to find out the nature and intensity of stressors perceived by the mid level undergraduate medical students (phase II and III) of Armed Forces Medical College before summative examination. The ultimate objective was to create a scope of shifting unfavourable stressor to favourable one.

\section{Materials and Method}

A prospective cross sectional descriptive study was designed involving the cadets of Armed Forces Medical College. Ethical clearance was taken from the authority submitting a protocol. The study was conducted during the period January to June 2018. Regularly passed students of phase II and III were included in the study and the response was collected in the first week of April 2018 ( 3 weeks prior to beginning of summative examination). The purpose and the process of data collection were explained to the students and a structured questionnaire was distributed to the volunteers of target population only. The foreign students were excluded from the study. Validated structured set of questionnaire (Medical Student Stressor Questionnaire -MSSQ) ${ }^{1}$ was selected for the study. For better and easy understanding questions were translated into bangla and were slightly modified for better adaptation to the topic of study. Collected data was analyzed in SPSS 19.

\section{Result}

Students of both phases opined that of the academic related stressors (Table-1) all factors were close to moderate in nature and some factors were even high in nature (means ranged from 1.93 to 3.18 of phase II and 1.88 to 2.74 of phase III). The maximum stress was for 'falling behind in reading schedule' and was closely followed by 'getting poor mark' as well as 'not enough scope of medical skill practice'. Students of phase II were in significantly higher stress $(p<0.00)$.

Bangladesh Journal of Medical Education 2018;9(2):3-10 
Table 1 : Frequency distribution of Academic Related Stressor (ARS) with their mean scores ( $\mathrm{n}=74$ for Phase II and 43 for Phase III)

\begin{tabular}{|c|c|c|c|c|c|c|c|c|}
\hline \multirow[b]{2}{*}{$\begin{array}{l}\text { Factors (at which level following } \\
\text { causes stress in you) }\end{array}$} & \multirow[b]{2}{*}{ Phase } & \multicolumn{5}{|c|}{ Level of stress (Score 0 to 4) } & \multirow[b]{2}{*}{$\begin{array}{c}\text { Score } \\
\text { Mean } \pm \text { SD }\end{array}$} & \multirow[b]{2}{*}{$\mathbf{p}$} \\
\hline & & No $f(\%)$ & $\begin{array}{c}\mathrm{M} \\
\text { ild } \mathrm{f}(\%)\end{array}$ & $\begin{array}{c}\text { Moderate } \\
\text { f (\%) }\end{array}$ & $\begin{array}{l}\text { High } \\
\text { f (\%) }\end{array}$ & $\begin{array}{l}\text { Severe } \\
\text { f }(\%)\end{array}$ & & \\
\hline \multirow[t]{2}{*}{ Tests/examinations } & $\mathrm{II}(\mathrm{n}=73)$ & $1(1.4)$ & $21(29.2)$ & $24(33.3)$ & $14(19.4)$ & $12(16.7)$ & $2.18 \pm 1.11$ & \multirow{2}{*}{0.23} \\
\hline & III & $4(9.3)$ & $8(18.6)$ & $21(48.8)$ & $7(16.3)$ & $3(7.0)$ & $1.93 \pm 1.0$ & \\
\hline \multirow[t]{2}{*}{ Falling behind in reading schedule } & II $(n=73)$ & $1(1.4)$ & $5(6.8)$ & $5(6.8)$ & $31(42.5)$ & $31(42.5)$ & $3.18 \pm 0.93$ & \multirow{2}{*}{$0.02^{*}$} \\
\hline & III & $2(4.7)$ & $4(9.3)$ & $8(18.6)$ & $18(41.9)$ & $11(25.6)$ & $2.74 \pm 1.1$ & \\
\hline \multirow[t]{2}{*}{ Large amount of content to be learnt } & II & $0(00)$ & $7(9.5)$ & $20(27.0)$ & $33(44.6)$ & $14(18.9)$ & $2.73 \pm 0.88$ & \multirow{2}{*}{0.07} \\
\hline & III & $1(2.3)$ & $9(20.9)$ & $11(25.6)$ & $16(37.2)$ & $6(14.0)$ & $2.40 \pm 1.05$ & \\
\hline \multirow{2}{*}{$\begin{array}{l}\text { Having difficulty in under-standing the } \\
\text { content }\end{array}$} & $\mathrm{II}(\mathrm{n}=72)$ & $4(5.6)$ & $10(13.9)$ & $15(20.8)$ & $30(41.7)$ & $13(18.1)$ & $2.53 \pm 1.11$ & \multirow{2}{*}{0.33} \\
\hline & III & $0(0.0)$ & $11(25.6)$ & $12(27.9)$ & $15(34.9)$ & $5(11.6)$ & $2.33 \pm 1.0$ & \\
\hline \multirow[t]{2}{*}{ Getting poor marks } & II $(n=73)$ & $4(5.6)$ & $2(13.9)$ & $19(20.8)$ & $12(31.7)$ & $36(18.1)$ & $3.01 \pm 1.17$ & \multirow{2}{*}{$0.01 *$} \\
\hline & III & $2(4.7)$ & $6(14.0)$ & $13(30.2)$ & $15(34.9)$ & $7(16.3)$ & $2.44 \pm 1.08$ & \\
\hline \multirow[t]{2}{*}{ Biasness examiner in evaluation } & $\mathrm{II}(\mathrm{n}=73)$ & $5(6.8)$ & $11(15.1)$ & $11(15.1)$ & $13(17.8)$ & $33(45.2)$ & $2.79 \pm 1.34$ & \multirow{2}{*}{$0.00^{*}$} \\
\hline & III & $8(18.6)$ & $7(16.3)$ & $12(27.9)$ & $9(20.9)$ & $7(16.3)$ & $2.0 \pm 1.35$ & \\
\hline \multirow{2}{*}{$\begin{array}{l}\text { Lack of time to review what have been } \\
\text { learnt }\end{array}$} & II & $4(5.4)$ & $8(10.8)$ & $24(32.4)$ & $18(24.3)$ & $20(27.0)$ & $2.57 \pm 1.16$ & \multirow{2}{*}{0.27} \\
\hline & III & $1(2.3)$ & $11(25.6)$ & $10(23.3)$ & $15(34.9)$ & $6(14.0)$ & $2.33 \pm 1.1$ & \\
\hline \multirow[t]{2}{*}{ Need to do well (self-expectation) } & II & $7(9.5)$ & $19(25.7)$ & $24(32.4)$ & $15(20.3)$ & $9(12.2)$ & $2.00 \pm 1.16$ & \multirow{2}{*}{0.84} \\
\hline & III & $5(11.6)$ & $9(20.9)$ & $15(34.9)$ & $7(16.3)$ & $7(16.3)$ & $2.05 \pm 1.23$ & \\
\hline \multirow[t]{2}{*}{ Learning context is full of competition } & II $(\mathrm{n}=73)$ & $3(4.1)$ & $8(11.0)$ & $18(24.7)$ & $19(26.0)$ & $25(34.2)$ & $2.75 \pm 1.16$ & \multirow{2}{*}{0.16} \\
\hline & III & $3(7.0)$ & $5(11.6)$ & $13(30.2)$ & $14(32.6)$ & $8(18.6)$ & $2.44 \pm 1.14$ & \\
\hline \multirow{2}{*}{$\begin{array}{l}\text { Unable to answer the questions of the } \\
\text { teachers }\end{array}$} & II & $4(5.4)$ & $11(14.9)$ & $19(25.7)$ & $18(24.3)$ & $22(29.7)$ & $2.58 \pm 1.22$ & \multirow{2}{*}{0.20} \\
\hline & $\mathrm{III}(\mathrm{n}=42)$ & $4(9.5)$ & $5(11.9)$ & $18(42.9)$ & $12(28.6)$ & $3(7.1)$ & $2.12 \pm 1.04$ & \\
\hline \multirow[t]{2}{*}{ High workload } & $\mathrm{II}(\mathrm{n}=72)$ & $1(1.4)$ & $8(11.1)$ & $17(23.6)$ & $23(31.9)$ & $23(31.9)$ & $2.82 \pm 1.05$ & \multirow{2}{*}{$0.04 *$} \\
\hline & III & $1(2.3)$ & $10(23.3)$ & $10(23.3)$ & $15(34.9)$ & $7(16.3)$ & $2.40 \pm 1.09$ & \\
\hline \multirow[t]{2}{*}{ Unjustified grading process } & II & $19(25.7)$ & $10(13.5)$ & $18(24.3)$ & $11(14.9)$ & $16(21.6)$ & $1.93 \pm 1.48$ & \\
\hline & $\mathrm{III}(\mathrm{n}=41)$ & $8(19.5)$ & $10(24.4)$ & $8(19.5)$ & $9(22.0)$ & $6(14.6)$ & $1.88 \pm 1.36$ & 0.06 \\
\hline Not enough scope of medical skill & II & $2(2.7)$ & $7(9.5)$ & $18(24.3)$ & $20(27.0)$ & $27(36.5)$ & $2.85 \pm 1.11$ & $000 *$ \\
\hline & III & $4(9.3)$ & $8(18.6)$ & $10(23.3)$ & $15(34.9)$ & $6(14.0)$ & $2.26 \pm 1.2$ & $0.00^{\circ}$ \\
\hline Feeling guilt during rest & II & $20(27.0)$ & $13(17.6)$ & $8(10.8)$ & $18(24.3)$ & $15(20.3)$ & $1.93 \pm 1.53$ & \\
\hline & III & $9(20.9)$ & $7(16.3)$ & $16(37.2)$ & $5(11.0)$ & $6(14.0)$ & $1.81 \pm 1.3$ & $0.6 /$ \\
\hline Phase II : Mean $\pm S$ & $=2.56 \pm 1.24$ & & & & ase III : M & ean $\pm \mathrm{SD}=$ & $.22 \pm 1.165$ & \\
\hline Phase II $($ ANOVA $): \mathrm{df}=1$ & $=8.405 ; \mathrm{p}$ & $=0.000^{*}$ & & Phase II & (ANOVA) & $\mathrm{df}=13$ & $=2.27 ; p=$ & $06^{*}$ \\
\hline & Phase II & vs Phase & $\mathrm{t}=5.4$ & $\mathrm{p}=0.000^{*}$ & & & & \\
\hline
\end{tabular}

* Differences between means are statistically significant 
Table 2 : Frequency distribution of Group Activity Related Stressor (GARS) with their mean scores ( $\mathrm{n}=74$ for Phase II and 43 for Phase III)

\begin{tabular}{|c|c|c|c|c|c|c|c|c|}
\hline \multirow{2}{*}{$\begin{array}{l}\text { Factors (at which level following } \\
\text { causes stress in you) }\end{array}$} & \multirow[b]{2}{*}{ Phase } & \multicolumn{5}{|c|}{ Level of stress (Score 0 to 4) } & \multirow[b]{2}{*}{$\begin{array}{c}\text { Score } \\
\text { Mean } \pm \text { SD }\end{array}$} & \multirow[b]{2}{*}{$\mathbf{p}$} \\
\hline & & No f $(\%)$ & Mild f (\%) & $\begin{array}{l}\text { Moderate } \\
\text { f }(\%)\end{array}$ & $\begin{array}{l}\text { High } \\
\text { f }(\%)\end{array}$ & $\begin{array}{l}\text { Severe } \\
\text { f }(\%)\end{array}$ & & \\
\hline \multirow[t]{2}{*}{ Participation in class discussion } & II & $29(39.2)$ & $20(27.0)$ & $16(21.6)$ & $6(8.1)$ & $3(4.1)$ & $1.11 \pm 1.14$ & \multirow{2}{*}{0.97} \\
\hline & III & $16(37.2)$ & $13(30.2)$ & $9(20.9)$ & $3(7.0)$ & $2(4.7)$ & $1.12 \pm 1.14$ & \\
\hline \multirow[t]{2}{*}{ Participation in class presentation } & II & $16(21.6)$ & $22(29.7)$ & $18(24.3)$ & $12(26.2)$ & $6(8.1)$ & $1.59 \pm 1.23$ & \multirow{2}{*}{0.59} \\
\hline & III & $12(27.9)$ & $12(27.9)$ & $10(23.3)$ & $5(11.6)$ & $4(9.3)$ & $1.47 \pm 1.28$ & \\
\hline \multirow[t]{2}{*}{ Need to do well (imposed by others) } & $\mathrm{II}(\mathrm{n}=72)$ & $12(16.7)$ & $6(8.3)$ & $22(30.6)$ & $22(30.6)$ & $10(13.9)$ & $2.17 \pm 1.27$ & \multirow{2}{*}{0.20} \\
\hline & III & $5(11.6)$ & $14(32.6)$ & $9(20.9)$ & $12(27.9)$ & $3(7.0)$ & $1.86 \pm 1.17$ & \\
\hline \multirow[t]{2}{*}{ Feeling of incompetence $(n=42)$} & II & $3(4.1)$ & $10(13.5)$ & $9(12.2)$ & $21(28.4)$ & $31(41.9)$ & $2.91 \pm 1.21$ & \multirow{2}{*}{0.20} \\
\hline & $\mathrm{III}(\mathrm{n}=42)$ & $6(14.3)$ & $7(16.7)$ & $10(23.8)$ & $12(28.6)$ & $7(16.7)$ & $2.17 \pm 1.31$ & \\
\hline \multicolumn{4}{|c|}{ Phase II : Mean \pm SD $=1.94 \pm 1.38$} & \multicolumn{5}{|c|}{ Phase III : Mean $\pm \mathrm{SD}=1.65 \pm 1.276$} \\
\hline \multicolumn{4}{|c|}{ Phase II ( ANOVA) : df $=13 ; \mathrm{F}=30.162 ; \mathrm{p}=0.000^{*}$} & \multicolumn{5}{|c|}{ Phase III ( ANOVA) $: \mathrm{df}=13 ; \mathrm{F}=5.973 ; \mathrm{p}=0.001^{*}$} \\
\hline \multicolumn{9}{|c|}{ Phase II vs Phase III : $\mathrm{t}=2.269 ; \mathrm{p}=0.024^{*}$} \\
\hline
\end{tabular}

* Differences between means are statistically significant

Four factors of group activity related stressor (GARS) were considered and 'feeling of incompetence' was observed to be more stressful in students of either phase (Table-2). Phase II students were observed to be significantly in more stress $(\mathrm{p}<0.05)$.

Factors considered under drive and desire related stressor (DRS) were relatively mild stressors as expressed by the students of both phases (Table 3 ) and there was no significant difference $(p=0.14)$ of opinion of two phases. Of the teaching and learning related stressors (TLRS), 'not enough feedback from teachers', 'uncertainty of what is expected of students' and 'lack of recognition for work done by students' were identified as more than moderate stressor by the phase II students (Table 4). The stress was significantly less $(\mathrm{p}<0.00)$ in phase III students.

Table 3 : Frequency distribution of Drive \& Desire Related Stressor (DRS) with their mean scores ( $\mathrm{n}=74$ for Phase II and 43 for Phase III)

\begin{tabular}{|c|c|c|c|c|c|c|c|c|}
\hline \multirow{2}{*}{$\begin{array}{l}\text { Factors (at which level following } \\
\text { causes stress in you) }\end{array}$} & \multirow[b]{2}{*}{ Phase } & \multicolumn{5}{|c|}{ Level of stress (Score 0 to 4) } & \multirow{2}{*}{$\begin{array}{c}\text { Score } \\
\text { Mean } \pm \text { SD }\end{array}$} & \multirow[b]{2}{*}{$\mathbf{p}$} \\
\hline & & No $f(\%)$ & Mild f (\%) & $\begin{array}{l}\text { Moderate } \\
\text { f (\%) }\end{array}$ & $\begin{array}{l}\text { High } \\
\text { f }(\%)\end{array}$ & $\begin{array}{l}\text { Severe } \\
\text { f }(\%)\end{array}$ & & \\
\hline \multirow[t]{2}{*}{ Unwillingness to study medicine } & $\mathrm{II}(\mathrm{n}=73)$ & $43(58.9)$ & $9(12.3)$ & $9(12.3)$ & $4(5.5)$ & $8(11.0)$ & $0.97 \pm 1.39$ & \multirow{2}{*}{0.20} \\
\hline & III & $27(64.3)$ & $7(16.7)$ & $4(9.5)$ & $2(4.8)$ & $2(4.8)$ & $0.69 \pm 1.14$ & \\
\hline \multirow[t]{2}{*}{ Parental wish to study medicine } & $\mathrm{II}(\mathrm{n}=73)$ & $39(53.4)$ & $11(15.1)$ & $8(11.0)$ & $7(9.6)$ & $8(11.0)$ & $1.1 \pm 1.43$ & \multirow{2}{*}{0.20} \\
\hline & III & $26(61.9)$ & $5(11.9)$ & $6(14.3)$ & $1(2.4)$ & $4(9.5)$ & $0.86 \pm 1.32$ & \\
\hline \multirow[t]{2}{*}{ Family responsibilities } & II & $17(23.0)$ & $7(9.5)$ & $18(24.3)$ & $12(16.2)$ & $20(27.0)$ & $2.15 \pm 1.51$ & \multirow{2}{*}{0.19} \\
\hline & III & $8(19.0)$ & $6(14.3)$ & $14(33.3)$ & $8(19.0)$ & $6(14.3)$ & $1.95 \pm 1.3$ & \\
\hline \multicolumn{4}{|c|}{ Phase II : Mean \pm SD $=1.41 \pm 1.531$} & \multicolumn{5}{|c|}{ Phase III : Mean \pm SD $=1.17 \pm 1.367$} \\
\hline \multicolumn{4}{|c|}{ Phase II ( ANOVA) $: \mathrm{df}=13 ; \mathrm{F}=14.785 ; \mathrm{p}=0.000^{*}$} & \multicolumn{5}{|c|}{ Phase III ( ANOVA $): \mathrm{df}=13 ; \mathrm{F}=12.513 ; \mathrm{p}=0.000^{*}$} \\
\hline \multicolumn{9}{|c|}{ Phase II vs Phase III $: \mathrm{t}=1.473 ; \mathrm{p}=0.142$} \\
\hline
\end{tabular}

* Differences between means are statistically significant 
Table 4 : Frequency distribution of Teaching and Learning Related Stressor (TLRS) with their mean scores ( $\mathrm{n}=74$ for Phase II and 43 for Phase III)

\begin{tabular}{|c|c|c|c|c|c|c|c|c|}
\hline \multirow{2}{*}{$\begin{array}{l}\text { Factors (at which level following } \\
\text { causes stress in you) }\end{array}$} & \multirow[b]{2}{*}{ Phase } & \multicolumn{5}{|c|}{ Level of stress (Score 0 to 4) } & \multirow{2}{*}{$\begin{array}{c}\text { Score } \\
\text { Mean } \pm \text { SD }\end{array}$} & \multirow[b]{2}{*}{$\mathbf{p}$} \\
\hline & & No $f(\%)$ & Mild f (\%) & $\begin{array}{l}\text { Moderate } \\
\text { f }(\%)\end{array}$ & $\begin{array}{l}\text { High } \\
\text { f }(\%)\end{array}$ & $\begin{array}{l}\text { Severe } \\
\text { f }(\%)\end{array}$ & & \\
\hline \multirow[t]{2}{*}{ Teacher with lack of teaching skills } & II & $15(20.3)$ & $17(23.0)$ & $20(27.0)$ & $11(14.9)$ & $11(14.9)$ & $1.81 \pm 1.33$ & \multirow{2}{*}{0.20} \\
\hline & $\operatorname{III}(\mathrm{n}=42)$ & $12(28.6)$ & $11(26.2)$ & $8(19.0)$ & $8(19.0)$ & $3(7.1)$ & $1.50 \pm 1.3$ & \\
\hline \multirow[t]{2}{*}{ Not having enough study material } & II & $5(6.8)$ & $14(18.9)$ & $28(37.8)$ & $14(18.9)$ & $13(17.6)$ & $2.22 \pm 1.15$ & \multirow{2}{*}{$0.00^{*}$} \\
\hline & III & $9(20.9)$ & $11(25.6)$ & $14(32.6)$ & $7(16.3)$ & $2(4.7)$ & $1.58 \pm 1.14$ & \\
\hline \multirow[t]{2}{*}{ Inappropriate assignments } & $\mathrm{II}(\mathrm{n}=73)$ & 1013.7() & $10(13.7)$ & $22(30.1)$ & $18(24.7)$ & $13(17.8)$ & $2.19 \pm 1.28$ & \multirow{2}{*}{$0.05^{*}$} \\
\hline & III & $9(20.9)$ & $8(18.6)$ & $15(34.9)$ & $8(18.6)$ & $3(7.0)$ & $1.72 \pm 1.2$ & \\
\hline \multirow[t]{2}{*}{ Lack of guidance from teacher(s) } & II $(n=42)$ & $7(9.5)$ & 11(14.9) & $16(21.6)$ & $24(32.4)$ & $16(21.6)$ & $2.42 \pm 1.25$ & \multirow{2}{*}{0.20} \\
\hline & III & $10(23.8)$ & $5(11.9)$ & $15(35.7)$ & $6(14.3)$ & $6(14.3)$ & $1.83 \pm 1.34$ & \\
\hline \multirow{2}{*}{$\begin{array}{l}\text { Not enough feedback from teacher(s) } \\
(\mathrm{n}=42)\end{array}$} & II $(n=73)$ & $5(6.8)$ & $11(15.1)$ & $12(16.4)$ & $17(23.3)$ & $28(38.4)$ & $2.71 \pm 1.31$ & \multirow{2}{*}{0.20} \\
\hline & III & $4(9.5)$ & $11(26.2)$ & $12(28.6)$ & $10(23.8)$ & $5(11.9)$ & $2.02 \pm 1.18$ & \\
\hline \multirow{2}{*}{$\begin{array}{l}\text { Uncertainty of what is expected of } \\
\text { students }\end{array}$} & II & $1(1.4)$ & $6(8.1)$ & $17(23.0)$ & $19(25.7)$ & $31(41.9)$ & $2.99 \pm 1.05$ & \multirow{2}{*}{$0.00 *$} \\
\hline & III & $2(4.7)$ & $9(20.9)$ & $17(39.5)$ & $9(20.9)$ & $6(14.0)$ & $2.19 \pm 1.08$ & \\
\hline \multirow{2}{*}{$\begin{array}{l}\text { Lack of recognition for work done by } \\
\text { students }\end{array}$} & II & $9(12.2)$ & $5(6.8)$ & $16(21.6)$ & $19(25.7)$ & $25(33.8)$ & $2.62 \pm 1.34$ & \multirow{2}{*}{$0.02 *$} \\
\hline & III & $7(16.3)$ & $9(20.9)$ & $10(23.3)$ & $9(20.9)$ & $8(18.6)$ & $2.05 \pm 1.36$ & \\
\hline \multicolumn{4}{|c|}{ Phase II : Mean $\pm \mathrm{SD}=2.42 \pm 1.292$} & \multicolumn{5}{|c|}{ Phase III : Mean \pm SD $=1.84 \pm 1.241$} \\
\hline \multicolumn{4}{|c|}{ Phase II (ANOVA) $: \mathrm{df}=13 ; \mathrm{F}=7.206 ; \mathrm{p}=0.000^{*}$} & \multicolumn{5}{|c|}{ Phase III (ANOVA) $: \mathrm{df}=13 ; \mathrm{F}=1.842 ; \mathrm{p}=0.091$} \\
\hline \multicolumn{9}{|c|}{ Phase II vs Phase III : $\mathrm{t}=6.26 ; \mathrm{p}=0.000^{*}$} \\
\hline
\end{tabular}

* Differences between means are statistically significant

Table 5 : Frequency distribution of Intra- \& Inter-personnel Related Stressor (IRS) with their mean scores (n=74 for Phase II and 43 for Phase III)

\begin{tabular}{|c|c|c|c|c|c|c|c|c|}
\hline \multirow{2}{*}{$\begin{array}{l}\text { Factors (at which level following } \\
\text { causes stress in you) }\end{array}$} & \multirow[b]{2}{*}{ Phase } & \multicolumn{5}{|c|}{ Level of stress (Score 0 to 4) } & \multirow[b]{2}{*}{$\begin{array}{c}\text { Score } \\
\text { Mean } \pm \text { SD }\end{array}$} & \multirow[b]{2}{*}{ p } \\
\hline & & No $f(\%)$ & Mild f (\%) & $\begin{array}{l}\text { Moderate } \\
\text { f }(\%)\end{array}$ & $\begin{array}{l}\text { High } \\
\text { f (\%) }\end{array}$ & $\begin{array}{l}\text { Severe } \\
\text { f }(\%)\end{array}$ & & \\
\hline \multirow[t]{2}{*}{ Conflicts with other students } & II & $11(14.9)$ & $14(18.9)$ & $9(12.2)$ & $19(25.7)$ & $21(28.4)$ & $2.34 \pm 1.45$ & \multirow{2}{*}{$0.00^{*}$} \\
\hline & III & $16(37.2)$ & $7(16.3)$ & $9(20.9)$ & $8(18.6)$ & $3(7.0)$ & $1.42 \pm 1.35$ & \\
\hline \multirow[t]{2}{*}{ Poor motivation to learn } & II $(\mathrm{n}=73)$ & $4(5.5)$ & $5(6.8)$ & $20(27.4)$ & $25(34.2)$ & $19(26.0)$ & $2.68 \pm 1.10$ & \multirow{2}{*}{$0.00^{*}$} \\
\hline & III & $9(20.9)$ & $9(20.9)$ & $9(20.9)$ & $12(27.9)$ & $4(9.3)$ & $1.84 \pm 1.31$ & \\
\hline \multirow{2}{*}{$\begin{array}{l}\text { Verbal or physical abuse by other } \\
\text { student(s) }\end{array}$} & II & 11(14.9) & 11(14.9) & $10(13.5)$ & $19(25.7)$ & $23(31.1)$ & $2.43 \pm 1.44$ & \multirow{2}{*}{$0.01 *$} \\
\hline & III & $15(34.9)$ & $4(9.3)$ & $6(14.0)$ & $13(30.2)$ & $5(11.6)$ & $1.74 \pm 1.5$ & \\
\hline \multirow[t]{2}{*}{ Verbal or physical abuse by teacher(s) } & II & $11(14.9)$ & $5(6.8)$ & $9(12.2)$ & $17(23.0)$ & $32(43.2)$ & $2.73 \pm 1.46$ & \multirow{2}{*}{0.06} \\
\hline & $\operatorname{III}(\mathrm{n}=41)$ & $13(31.7)$ & $5(12.2)$ & $8(19.5)$ & $10(24.4)$ & $5(12.2)$ & $1.73 \pm 1.45$ & \\
\hline \multirow[t]{2}{*}{ Verbal or physical abuse by personnel(s) } & II & $14(18.9)$ & $5(6.8)$ & $14(18.9)$ & $12(16.2)$ & $29(39.2)$ & $2.50 \pm 1.53$ & \multirow{2}{*}{0.20} \\
\hline & $\operatorname{III}(n=42)$ & $13(31.0)$ & $2(4.8)$ & $11(26.2)$ & $10(23.8)$ & $6(14.3)$ & $1.86 \pm 1.46$ & \\
\hline \multirow[t]{2}{*}{ Conflict with personnel(s) } & II & $10(13.5)$ & $4(5.4)$ & $13(17.6)$ & $24(32.4)$ & $23(31.1)$ & $2.62 \pm 1.34$ & \multirow{2}{*}{$0.00 *$} \\
\hline & III & $16(37.2)$ & $3(7.0)$ & $7(16.3)$ & $12(27.9)$ & $5(11.6)$ & $1.70 \pm 1.51$ & \\
\hline \multirow[t]{2}{*}{ Conflict with teacher(s) } & II & $9(12.2)$ & $5(6.8)$ & $4(5.4)$ & $7(9.5)$ & $49(66.2)$ & $3.11 \pm 1.45$ & \multirow{2}{*}{$0.00^{*}$} \\
\hline & III & $17(39.5)$ & $3(7.0)$ & $5(11.6)$ & $10(23.3)$ & $8(18.6)$ & $1.74 \pm 1.62$ & \\
\hline \multicolumn{4}{|c|}{ Phase II : Mean $\pm \mathrm{SD}=2.63 \pm 1.413$} & \multicolumn{5}{|c|}{ Phase III : Mean \pm SD $=1.72 \pm 1.450$} \\
\hline \multicolumn{4}{|c|}{ Phase II ( ANOVA) : $\mathrm{df}=13 ; \mathrm{F}=2.403 ; \mathrm{p}=0.027^{*}$} & \multicolumn{5}{|c|}{ Phase III ( ANOVA) $: \mathrm{df}=13 ; \mathrm{F}=0.42 ; \mathrm{p}=0.865$} \\
\hline
\end{tabular}

* Differences between means are statistically significant

Bangladesh Journal of Medical Education 2018;9(2):3-10 
Students of phase III opined that factors of intra- and interpersonnel related stressor (IRS) were less than moderate stressor (Table 5); whereas students of phase II identified 'conflict with the teacher' as high stressor. Of the social related stress (SRS) factors, students of both phases expressed that 'facing illness or death of the patients' and 'army environment/too much restriction in campus' produced high stress in them (Table 6).

Table 6 : Frequency distribution of Social Related Stressor (SRS) with their mean scores (n=74 for Phase II and 43 for Phase III)

\begin{tabular}{|c|c|c|c|c|c|c|c|c|}
\hline \multirow{2}{*}{$\begin{array}{l}\text { Factors (at which level following } \\
\text { causes stress in you) }\end{array}$} & \multirow[b]{2}{*}{ Phase } & \multicolumn{5}{|c|}{ Level of stress (Score 0 to 4) } & \multirow[b]{2}{*}{$\begin{array}{c}\text { Score } \\
\text { Mean } \pm \text { SD }\end{array}$} & \multirow[b]{2}{*}{$\mathbf{p}$} \\
\hline & & No f $(\%)$ & Mild f (\%) & $\begin{array}{l}\text { Moderate } \\
\text { f (\%) }\end{array}$ & $\begin{array}{l}\text { High } \\
\text { f }(\%)\end{array}$ & $\begin{array}{l}\text { Severe } \\
\text { f }(\%)\end{array}$ & & \\
\hline \multirow[t]{2}{*}{ Lack of time for family and friends } & II & $11(14.9)$ & $11(14.9)$ & $24(32.4)$ & $9(12.2)$ & $19(25.7)$ & $2.19 \pm 1.37$ & \multirow{2}{*}{0.50} \\
\hline & III & $3(7.0)$ & $14(32.6)$ & $10(23.3)$ & $11(25.6)$ & $5(11.6)$ & $2.02 \pm 1.17$ & \\
\hline \multirow{2}{*}{$\begin{array}{l}\text { Unable to answer questions from } \\
\text { patients }\end{array}$} & II $(n=73)$ & $7(9.5)$ & $11(14.9)$ & $16(21.6)$ & $25(33.8)$ & $15(20.3)$ & $2.41 \pm 1.24$ & \multirow{2}{*}{0.20} \\
\hline & III & $7(16.3)$ & $6(14.0)$ & $12(27.9)$ & $12(27.9)$ & $6(14.0)$ & $2.09 \pm 1.29$ & \\
\hline \multirow{2}{*}{$\begin{array}{l}\text { Talking to patients about personal } \\
\text { problems }\end{array}$} & II & $17(23.6)$ & $9(12.5)$ & $20(27.8)$ & $16(22.2)$ & $10(13.9)$ & $1.90 \pm 1.37$ & \multirow{2}{*}{0.81} \\
\hline & III & $15(34.9)$ & $4(9.3)$ & $5(11.6)$ & $11(25.6)$ & $8(18.6)$ & $1.84 \pm 1.59$ & \\
\hline \multirow[t]{2}{*}{ Facing illness or death of the patients } & II & $7(9.5)$ & $3(4.1)$ & $7(9.5)$ & $11(14.9)$ & $46(62.2)$ & $3.16 \pm 1.31$ & \multirow{2}{*}{0.19} \\
\hline & $\operatorname{III}(n=41)$ & $4(9.5)$ & $3(7.1)$ & $1(2.4)$ & $12(28.6)$ & $22(52.4)$ & $3.07 \pm 1.31$ & \\
\hline \multirow[t]{2}{*}{ Working with computers } & II & $25(34.2)$ & $18(24.7)$ & $17(23.3)$ & $8(11.0)$ & $5(6.8)$ & $1.32 \pm 1.25$ & \multirow{2}{*}{0.19} \\
\hline & $\operatorname{III}(\mathrm{n}=42)$ & $16(38.1)$ & $7(16.7)$ & $10(23.8)$ & $5(11.9)$ & $4(9.5)$ & $1.38 \pm 1.36$ & \\
\hline \multirow{2}{*}{$\begin{array}{l}\text { Frequent interruption of my work by } \\
\text { others }\end{array}$} & II & $2(2.7)$ & 13(17.6) & $14(18.9)$ & $31(41.9)$ & $14(18.9)$ & $2.57 \pm 1.07$ & \multirow{2}{*}{0.38} \\
\hline & III & $8(18.6)$ & $5(11.6)$ & $12(27.9)$ & $11(25.6)$ & $7(16.3)$ & $2.09 \pm 1.34$ & \\
\hline \multirow[t]{2}{*}{ Too much restriction in campus } & II & $4(5.4)$ & $2(2.7)$ & $5(6.8)$ & $9(12.2)$ & $54(73.0)$ & $3.45 \pm 1.10$ & \multirow{2}{*}{0.26} \\
\hline & III & $3(7.0)$ & $1(2.3)$ & $10(23.3)$ & $10(23.3)$ & $19(44.2)$ & $2.95 \pm 1.19$ & \\
\hline \multicolumn{4}{|c|}{ Phase II : Mean \pm SD $=2.43 \pm 1.412$} & \multicolumn{5}{|c|}{ Phase III : Mean $\pm \mathrm{SD}=2.21 \pm 1.427$} \\
\hline \multicolumn{4}{|c|}{ Phase II ( ANOVA) $: \mathrm{df}=13 ; \mathrm{F}=24.867 ; \mathrm{p}=0.000^{*}$} & \multicolumn{5}{|c|}{ Phase III ( ANOVA) $: \mathrm{df}=13 ; \mathrm{F}=8.782 ; \mathrm{p}=0.000^{*}$} \\
\hline \multicolumn{9}{|c|}{ Phase II vs Phase III : $t=2.171 ; p=0.03^{*}$} \\
\hline
\end{tabular}

* Differences between means are statistically significant

Of the six groups of stressors considered in this study, academic related stressor (ARS), intra- \& inter-personnel related stressor (IIRS), social related stressor (SRS) and teaching \& learning related stressor (TLRS) were revealed to be significantly more $(p=0.000)$ intense in nature in phase II students (table 7). Among the students of phase III, academic related stressor (ARS) and social related stressor (SRS) were significantly more $(\mathrm{p}=0.000)$ intense in nature (table 8$)$.
Table 7 : Analysis of variance of different types of level of stressors of Phase II students $(\mathrm{n}=74$

\begin{tabular}{|c|c|c|c|}
\hline Type of Stressor & $\begin{array}{c}\text { Score } \\
\text { Mean } \pm \text { SD }\end{array}$ & $\mathbf{F}$ & $\mathbf{p}$ \\
\hline $\begin{array}{l}\text { Academic Related Stress } \\
\text { (ARS) }\end{array}$ & $2.56 \pm 1.241$ & \multirow{6}{*}{$\begin{array}{l}36.853 \\
(\mathrm{df}=5)\end{array}$} & \multirow{6}{*}{$0.000 *$} \\
\hline $\begin{array}{l}\text { Group Activity Related } \\
\text { Stressor (GARS) }\end{array}$ & $1.94 \pm 1.38$ & & \\
\hline $\begin{array}{l}\text { Drive \& Desire Related } \\
\text { Stressor (DRS) }\end{array}$ & $1.41 \pm 1.531$ & & \\
\hline $\begin{array}{l}\text { Teaching \& Learning Related } \\
\text { Stressor (TLRS) }\end{array}$ & $2.42 \pm 1.292$ & & \\
\hline $\begin{array}{l}\text { Intra- \& Inter-personnel } \\
\text { Related Stressor (IIRS) }\end{array}$ & $2.63 \pm 1.413$ & & \\
\hline Social Related Stressor (SRS) & $2.43 \pm 1.412$ & & \\
\hline
\end{tabular}

* Difference between means is statistically significant 
Table 8 : Analysis of variance of different types of level of stressors of Phase III students ( $\mathrm{n}=43$ )

\begin{tabular}{|l|c|c|c|}
\hline Type of Stressor & $\begin{array}{c}\text { Score } \\
\text { Mean } \pm \text { SD }\end{array}$ & F & p \\
\cline { 1 - 2 } $\begin{array}{l}\text { Academic Related Stress } \\
\text { (ARS) }\end{array}$ & $2.22 \pm 1.165$ & & \\
\cline { 1 - 2 } $\begin{array}{l}\text { Group Activity Related } \\
\text { Stressor (GARS) }\end{array}$ & $1.65 \pm 1.276$ & & \\
\cline { 1 - 2 } $\begin{array}{l}\text { Drive \& Desire Related } \\
\text { Stressor (DRS) }\end{array}$ & $1.17 \pm 1.367$ & \multirow{2}{*}{$0.000^{*}$} \\
\cline { 1 - 2 } $\begin{array}{l}\text { Teaching \& Learning Related } \\
\text { Stressor (TLRS) }\end{array}$ & $1.84 \pm 1.241$ & \\
\cline { 1 - 2 } $\begin{array}{l}\text { Intra- \& Inter-personnel } \\
\text { Related Stressor (IIRS) }\end{array}$ & $1.72 \pm 1.450$ & & \\
\cline { 1 - 2 } Social Related Stressor (SRS) & $2.21 \pm 1.427$ & & \\
\hline
\end{tabular}

*Difference between means is statistically significant

\section{Discussion}

All types of courses are stressful. Study showed that medical students had higher stress level compared to the engineering, arts as well as to commerce students ${ }^{4}$. To workout the level and type of stresses in students various questionnaires and scales were used by researches keeping in mind the socioeconomic demography ${ }^{2-5}$. The target population of this study was students of a full residential medical college where a uniform standard of living and social security exist. Yusoff et al developed the Medical Student Stressor Questionnaire (MSSQ) reviewing various literature of the subject and discussing with experts working in the field. Researcher determined the construct validity and the internal consistency of that questionnaire (MSSQ) and observed to have good psychometric value to identify stressors among medical students ${ }^{1}$. This questionnaire was used in this study with very little modification.

Present study revealed that students of both phases experience academic related stressors tobe similar in nature; but intensity was significantly $(\mathrm{p}=0.000)$ higher in phase II students. Of the factors, 'falling behind in reading schedule', 'getting poor marks' and 'high workload' were reported as more stressful. These were revealed as more stressful than 'test/examination'. Study conducted at Armed Forces Medical College, Pune observed that of this domain the leading causes of stress were 'need to do well in examination' and 'unjustified grading process' ${ }^{\prime 6}$. Study on 3rd year undergraduate medical students of Combined Military Hospital, Lahore Medical College revealed 'high parental expectations', 'frequency of examinations' and 'vastness of academic curriculum', as the most frequently and severely occurring sources of stress? ${ }^{7}$. In a study conducted at University of Washington Medical College, Vitaliano et al observed peer completion as one of the major stressor ${ }^{8}$. The regional variation is very much expected.

This study showed that group activity as well as desire related stressors were less intense in nature. At the mid-level part of the course, the students usually cope with this type of stressors. Mehrotra and Devarakonda also observed these two domains as least stressor area ${ }^{6}$. The interpersonal and teaching-learning related stressors were observed to be more prominent in phase II students. This may be related to the behaviour of a particular teacher of that phase.

After completion of phase I the students are exposed to hospital environment and started observing closely the sufferings of sick person close. This has definite psychological impact on the students and the respondents of this study identified 'facing illness or death of the patients' as high stressor. Living in a restricted campus was also identified as high stressor. Staying in hostel was also opined as high stressor by the undergraduate medical students of Manipal College of Medical Sciences, Pokhara, Nepal'.

Intensity of response to various stressors was observed to be less among the phase III students than phase II. The mean scores observed in this study were close to those of the study of Mehrotra and Devarakonda ${ }^{6}$ and a beat different from study of Bansal and Pundir ${ }^{10}$. The regional and even institutional nature may affect stressor in a different way.

\section{Conclusion}

The objective of medical colleges is to produce competent doctors who will be acceptable to the society. This study focused the present status so that Stakeholders concentrate on the matter and incorporate strategies to improve the teaching, learning, evaluation and educational environment and help the students to develop stress coping skills in early medical career in order to reduce negative effects of stresses on the future doctors.

\section{References}

1. Yusoff MSB, Rahim AFA, Yaacob MJ. The development and validity of the medical student stressor questionnaire (MSSQ). ASEAN Journal of Psychiatry 2010 January-June 2010, 11 (1): 13-24.

2. Simran G, Sangeeta N, Lily W. Evaluation of Examination Anxiety Status and its Associated Factors Among First Professional Medical (MBBS) Students. International Journal of Interdisciplinary and Multidisciplinary Studies (IJIMS) 2015;2(8) : 1-11.

3. Bangladesh Medical and Dental Council. Curriculum for Undergraduate Medical Students. Dhaka : 2012.

4. Jafri SAM, Zaidi E, Aamir IS, Aziz HW, Imad-ud-Din, Shah MAH. Stress Level Comparison of Medical and Non-medical Students: A Cross Sectional Study done at Various Professional Colleges in Karachi, Pakistan. Acta Psychopathologica (iMedPub Journals) 2017; 3(2): 8. Available at: www.psychopathology.imedpub.com

5. Dahlin M, Joneborg N, Runeson B. Stress and depression among medical students : a cross-sectional study. Medical Education 2005; 39: 594-604.

6. Mehrotra S, Devarakonda S. Stress in undergraduate 
medical education: a cross-sectional study. International Journal of Recent Scientific Research 2015; 6(4): 3497-3500.

7. Shah M, Hasan S, Malik S, Sreeramareddy CT. Perceived Stress, Sources and Severity of Stress among medical undergraduates in a Pakistani Medical School. BMC Medical Education 2010, 10:2, 1-8.

8. Vitaliano PP, Russo J, Carr JE, Heerwagen JH. Medical School Pressures and their Relation to Anxiety. The Journal of Nervous and mental Disease 1984; 172(12): 730-6.
9. Sreeramareddy CT, Shankar PR, Binu VS, Mukhopadhyay C, Ray B, Menezes RG. Psychological morbidity, sources of stress and coping strategies among undergraduate medical students of Nepal. BMC Medical Education 2007, 7:26, 1-8. (doi:10.1186/ 1472-6920-7-26).

10. Bansal I, Pundir P. Stress among Early and Late Entrants in Medical Education. The International Journal of Indian Psychology. 2015 Oct-Dec; 3(1) 6, DIP: C00392V3I12015 